\title{
Platonic polyhedra tune the 3-sphere: III. Harmonic analysis on octahedral spherical 3-manifolds.
}

\author{
Peter Kramer, \\ Institut für Theoretische Physik der Universität Tübingen, \\ Germany.
}

August 17, 2018

\begin{abstract}
.
From the homotopy groups of three distinct octahedral spherical 3-manifolds we construct the isomorphic groups $H$ of deck transformations acting on the 3 -sphere. The $H$-invariant polynomials on the 3 -sphere constructed by representation theory span the bases for the harmonic analysis on three spherical manifolds. Analysis of the Cosmic Microwave Background in terms of these new bases can reveal a non-simple topology of the space part of space-time.
\end{abstract}

\section{Introduction.}

We view a spherical topological 3-manifold $\mathcal{M}$, see [12] and [14], as a prototile on its cover $\tilde{\mathcal{M}}=S^{3}$. We studied in [7] the isometric actions of $O(4, R)$ on the 3 sphere $S^{3}$ and gave its basis as well-known homogeneous Wigner polynomials in [5] eq.(37). An algorithm due to Everitt in [3] generates the homotopies for all spherical 3-manifolds $\mathcal{M}$ from five Platonic polyhedra. Using intermediate Coxeter groups, we construct deck transformations acting on the 3-sphere as isomorphic images [12] of homotopies and generate the groups $H=\operatorname{deck}(\mathcal{M}) \sim \pi_{1}(\mathcal{M})$. Following work on the Poincaré dodecahedral [5], 6], the tetrahedral [7], and two cubic spherical manifolds [8], we turn here to three octahedral spherical manifolds denoted in [3] as $N 4, N 5, N 6$. We construct a basis for the harmonic analysis on each manifold from $H$-invariant polynomials on the 3 -sphere.

One field of applications for harmonic analysis is cosmic topology [10], [11]: The topology of a 3-manifold $\mathcal{M}$ is favoured if data from the Cosmic Microwave Back- 
ground can be expanded in its harmonic basis. The present work provides three novel octahedral 3-manifolds for this analysis. For the notions of homotopic boundary conditions and random point symmetry we refer to [9].

\section{The Coxeter group $G$ and the 24-cell on $S^{3}$.}

The cartesian coordinates $x=\left(x_{0}, x_{1}, x_{2}, x_{3}\right) \in E^{4}$ for $S^{3}$ we combine as in [5], [7] in the matrix form

$$
u=\left[\begin{array}{ll}
z_{1} & z_{2} \\
-\bar{z}_{2} & \bar{z}_{1}
\end{array}\right], z_{1}=x_{0}-i x_{3}, z_{2}=-x_{2}-i x_{1}, z_{1} \bar{z}_{1}+z_{2} \bar{z}_{2}=1 .
$$

For the group action we start from the Coxeter group $G<O(4, R)$ [4], [3] p. 254, with the diagram

$$
G=: \circ \stackrel{3}{-} \circ \stackrel{4}{-} \circ \stackrel{3}{-} \circ .
$$

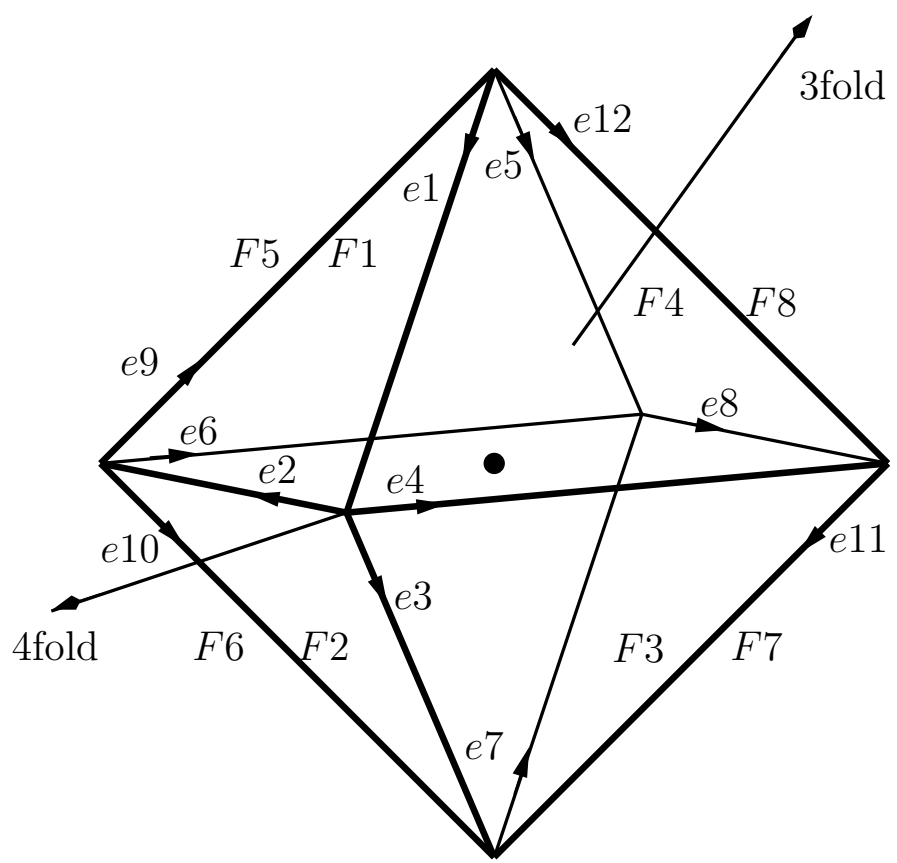

Fig. 1. The octahedron projected to the plane with faces $F 1 \ldots F 8$ and directed edges $e 1 \ldots e 12$ according to [3]. The products of Weyl reflections $\left(W_{1} W_{2}\right)$ and $\left(W_{2} W_{3}\right)$ generate right-handed 3 fold and 4 fold rotations respectively.

For the Coxeter diagram eq. 3 we give for the 4 Weyl reflections $W_{s}, s=1,2,3,4$ the Weyl vectors $a_{s}$ in Table 2.1 and compute for each $a_{s}=\left(a_{s 0}, a_{s 1}, a_{s 2}, a_{s 3}\right)$ the matrix

$$
v_{s}:=\left[\begin{array}{ll}
a_{s 0}-i a_{s 3} & -a_{s 2}-i a_{s 1} \\
a_{s 2}-i a_{s 1} & a_{s 0}+i a_{s 3}
\end{array}\right] \in S U(2, C) .
$$


The matrices $v_{s}$ are used to relate, see [7], the Weyl reflections to $\left(S U^{l}(2, C) \times\right.$ $S U^{r}(2, C)$ ) acting by left and right multiplication on the coordinates eq. 11. We include the (orientation preserving) inversion $J_{4} \in G$, and list the additional Weyl reflection $W_{0}$. The Coxeter group eq. 2 is of order $|G|=48 \times 24=1152$. The first three Weyl reflections from Table 2.1 generate, see [4], the cubic Coxeter subgroup

$$
O=: \circ \stackrel{4}{-} \circ \stackrel{3}{-} \circ
$$

isomorphic to the octahedral group $O \sim\left(C_{2}\right)^{3} \times_{s} S(3)$ acting on $E^{3} \in E^{4}$. The octahedral tiling of $S^{3}$ is the 24-cell discussed in [13] pp. 171-2. The center positions of the 24 octahedra in the octahedral 24-cell tiling are the midpoints of the 24 square faces of the 8 cubes in the 8-cell tiling shown in [8], Fig. 1. As shown in [13] pp. 178-9, vertices of six octahedra are located at each center of a cube from the 8-cell. Table 2.1 The Weyl vectors $a_{s}, s=1, . ., 4$ and $a_{0}$ for the Coxeter group $G$ eq. 2 , and the $2 \times 2$ unitary matrices $v_{s}$ eq. 3, in terms of $\theta:=\exp (i \pi / 4)$.

\begin{tabular}{|c|c|c|}
\hline$s$ & Weyl vector $a_{s}$ & matrix $v_{s}$ \\
\hline 1 & $\left(0, \sqrt{\frac{1}{2}},-\sqrt{\frac{1}{2}}, 0\right)$ & {$\left[\begin{array}{ll}0 & \bar{\theta} \\
-\theta & 0\end{array}\right]$} \\
\hline 2 & $\left(0,0,-\sqrt{\frac{1}{2}}, \sqrt{\frac{1}{2}}\right)$ & $\sqrt{\frac{1}{2}}\left[\begin{array}{ll}-i & 1 \\
-1 & i\end{array}\right]$ \\
\hline 3 & $(0,0,0,1)$ & $\begin{array}{ll}-i & 0 \\
0 & i\end{array}$ \\
\hline 4 & $\left(\frac{1}{2}, \frac{1}{2}, \frac{1}{2}, \frac{1}{2}\right)$ & $\sqrt{\frac{1}{2}}\left[\begin{array}{ll}\bar{\theta} & -\theta \\
\bar{\theta} & \theta\end{array}\right]$ \\
\hline 0 & $(1,0,0,0)$ & $\left|\begin{array}{ll}1 & 0 \\
0 & 1\end{array}\right|$ \\
\hline
\end{tabular}

\section{From homotopies to deck transformations.}

\subsection{Generators.}

The spherical Coxeter group $G$ is generated by the Weyl reflections $W_{s}$ given in Table 2.1. In the next section we give for the three octahedral manifolds $N 4, N 5, N 6$ the edge gluing schemes computed in [3], but include the corrections given in [1]. These corrections apply in particular to the manifold $N 5$. The construction proceeds in the following steps:

(i) An edge gluing scheme lists glued triples of oriented edges for pairs of glued faces $F i \cup F j$ in its rows. The four generators of the first homotopy group each prescribe a gluing of three oriented chains of edges, bounding counterclockwise a preimage face 
Fi and clockwise an image face Fj of the prototile. These chains taken from Fig. 1 are given between square brackets.

(ii) Any deck transformation is constructed from a homotopy by first rotating the preimage face $\mathrm{Fi}$ wrt. the center $(1,0,0,0)$ of the prototile to the position of face $\mathrm{F} 4$, and then applying a rotation $\left(W_{1} W_{2}\right)^{\nu}, \nu=0,1,2$ preserving the center of $\mathrm{F} 4$. Inversion $J_{3}$ in the center of the prototile then maps the preimage face from the position of face F4 to the one of F6. This inversion can be expressed as $J_{3}=$ $J_{4} W_{0}$. The total inversion $J_{4}$ preserves orientation and commutes with all rotations. Applying the Weyl reflection $W_{4}$, the preimage face now in position F6 is mapped into itself, while the octahedral prototile is mapped into an image tile.

(iii) By a final rotation wrt. the center of the prototile, the preimage face is mapped from the position of F6 into the image position of face Fj. An appropriate choice of $\nu$ yields the edge mapping prescribed by the homotopy. By virtue of the Weyl reflection, the image face $\mathrm{Fj}$ separates the prototile from a fixed octahedral image. The orientation of the chain of edges of the image face now is counterclockwise when referred to the center of the image tile. The map from the prototile to the image tile in this position is the deck transformation isomorphic to the homotopy.

All the operations in (i-iii) are elements of the Coxeter group $G$ and moreover of $S O(4, R)$. The rotations are generated from the 3fold rotation $\left(W_{1} W_{2}\right)$ and the 4 fold rotation $\left(W_{2} W_{3}\right)$, indicated in Fig. 1. Any Weyl reflection $W_{s}$ is associated with a $2 \times 2$ matrix $v_{s}$ given in Table 2.1. Products of two Weyl reflections generate rotations. The conversion from these products to rotations $g=\left(w_{l}, w_{r}\right)$ is given from [7] eq. (60) by

$$
\left(W_{i} W_{j}\right) \rightarrow T_{g}=T_{\left(w_{l}, w_{r}\right)}, g=\left(w_{l}, w_{r}\right)=\left(v_{i} v_{j}^{-1}, v_{i}^{-1} v_{j}\right) .
$$

The operator $T_{g}$ acts on functions $f(u)$ on $S^{3}$ in coordinates $u$ from eq. 1 as

$$
\left(T_{\left(w_{l}, w_{r}\right)} f\right)(u):=f\left(w_{l}^{-1} u w_{r}\right) .
$$

Any product of the in general five operations described under (i-iii) is a deck transformation, preserves orientation, and is isomorphic to a homotopic gluing. We list them for the three manifolds. Finally the deck transformations are converted by use of eq. 5, Table 2.1, and multiplication into pairs $\left(w_{l}, w_{r}\right) \in\left(S U^{r}(2, C) \times S U^{r}(2, C)\right)$, given in the following Tables.

The (isomorphic) groups $H$ of homotopies and of deck transformations, distinct for different manifolds, all have order 24 equal to the number of octahedral tiles. These groups if not abelian must appear in the Table of Coxeter and Moser [2] pp. 134-5. 


\subsection{Center positions under deck transformations.}

From the coordinates eq. 1 of $S^{3}$, the center $u=e$ of the octahedral prototile is transformed by $g=\left(w_{l}, w_{r}\right)$ into an image center

$$
g=\left(w_{l}, w_{r}\right) \in H: e \rightarrow u^{\prime}=w_{l}^{-1} w_{r} .
$$

Since all three groups of deck transformations must produce the same 24-cell, it follows that their lists of octahedral centers must coincide up to permutations. For the manifold $N 6$ we shall find that its group of deck transformations is the binary tetrahedral group $\mathcal{T}$ with all elements of the form $g=\left(w_{l}, e\right)$. From eq. 7 it then follows that the list of the 24 octahedral centers $u^{\prime}$ in the 24-cell can be written as

$$
N 6: g=\left(w_{l}, e\right) \in \mathcal{T}, u^{\prime}=w_{l}^{-1} .
$$

with $w_{l}$ running over all group elements in Table 6.2. The elements $g=\left(w_{l}, w_{r}\right)$ of the groups $H \neq \mathcal{T}$ for the manifolds $N 4, N 5$ therefore must reproduce by the products $u^{\prime}$ in eq. 7 these 24 center positions. For the manifold $N 5$ we display this relation in Table $\mathbf{5 . 2}$.

\subsection{Harmonic analysis on octahedral 3-manifolds.}

Once we have derived the explicit matrix form of the three groups $H$ of deck transformations, we have all the tools for the harmonic analysis. From any $g=\left(w_{l}, w_{r}\right) \in$ $S O(4, R)$ we can, as outlined in general in [7] eq.(44), pass to its representations $D^{(j, j)}(g)=D^{j}\left(w_{l}\right) \times D^{j}\left(w_{r}\right)$ by use of Wigner representation matrices $D^{j}$ of $S U(2, C)$. From these representations we can construct the general projection and Young operators [7] eq. (82) to $H$-invariant polynomials of fixed $j$ and degree $2 j$. The projection yields linear combinations of spherical harmonics or Wigner polynomials $D_{m_{1}, m_{2}}^{j}(u)$ of degree $2 j$. For the octahedral manifold $N 4$ we give the final result of this projection in Table 4.3. The characters follow from [7] eq. (45) and allow to derive by [7] eq.(62) the multiplicities for any degree $2 j$ and group $H$.

\section{Manifold N4}

Face gluings:

$$
F 6 \cup F 2, F 5 \cup F 3, F 1 \cup F 4, F 7 \cup F 8 .
$$

Edge gluing scheme:

$$
\left[\begin{array}{lll}
1 & 4 & 9 \\
2 & 7 & \overline{12} \\
3 & 6 & \overline{10} \\
5 & 8 & 11
\end{array}\right]
$$


Edge and face gluing generators of $\pi_{1}(N 4)$ :

$$
\begin{aligned}
& g_{1}: 6 \cup 2,\left[\begin{array}{lll}
\overline{10} & & \overline{7} \\
& 6 &
\end{array}\right] \rightarrow\left[\begin{array}{lll}
3 & & \overline{2} \\
& \overline{10} &
\end{array}\right], \\
& g_{2}: 5 \cup 3,\left[\begin{array}{lll}
5 & & 9 \\
& \overline{6} &
\end{array}\right] \rightarrow\left[\begin{array}{lll}
11 & & 4 \\
& \overline{3} &
\end{array}\right], \\
& g_{3}: 1 \cup 4,\left[\begin{array}{lll}
\overline{9} & & \overline{1} \\
& \overline{2} &
\end{array}\right] \rightarrow\left[\begin{array}{lll}
\overline{1} & & \overline{4} \\
& 12 &
\end{array}\right], \\
& g_{4}: 7 \cup 8,\left[\begin{array}{lll}
7 & & 11 \\
& 8 &
\end{array}\right] \rightarrow\left[\begin{array}{lll}
\overline{12} & & 8 \\
& 5 &
\end{array}\right],
\end{aligned}
$$

Isomorphic generators of $\operatorname{deck}(N 4)$ :

$$
\begin{aligned}
& g_{1}=\left(W_{2} W_{3}\right)^{2}\left(W_{1} W_{2}\right)\left(W_{4} W_{0}\right) J_{4} \\
& g_{2}=\left(W_{3} W_{2}\right)\left(W_{1} W_{2}\right)\left(W_{4} W_{0}\right)\left(W_{2} W_{3}\right) J_{4} \\
& g_{3}=\left(W_{2} W_{1}\right)\left(W_{4} W_{0}\right)\left(W_{2} W_{3}\right)^{2}\left(W_{2} W_{1}\right) J_{4} \\
& g_{4}=\left(W_{1} W_{2}\right)\left(W_{3} W_{2}\right)\left(W_{2} W_{1}\right)\left(W_{4} W_{0}\right)\left(W_{3} W_{2}\right) J_{4}
\end{aligned}
$$

Table 4.1 Generators $g=\left(w_{l}, w_{r}\right)$ of $\operatorname{deck}(N 4)$ in the scheme eqs. 5,6, We use the short-hand notation of Table 6.2.

\begin{tabular}{|l|l|l|}
\hline$g$ & $w_{l}$ & $w_{r}$ \\
\hline$g_{1}$ & $-\alpha_{2}$ & $\mu$ \\
\hline$g_{2}$ & $-\alpha_{2}^{-1}$ & $-e$ \\
\hline$g_{3}$ & $\alpha_{2}$ & $\nu$ \\
\hline$g_{4}$ & $\alpha_{2}^{-1}$ & $\omega$ \\
\hline
\end{tabular}

The generators $g=\left(w_{l}, w_{r}\right)$ have for $w_{l}$ the order 6 or 3 and $w_{l}^{3}= \pm e$, for $w_{r}$ the order 4 and $w_{r}^{2}=-e$. From this it follows that $g_{q}^{3}=\left( \pm e,-w_{r}\right) \sim\left(e, \pm w_{r}\right)$. It is easy to see that the four elements $g_{q}^{3}$ generate the quaternion group by right action which we denote by $Q^{r}$. Similarly the powers 4 of the generators fulfill $g_{q}^{4}=$ $\left(w_{l}^{4},-e\right) \sim\left(-w_{l}^{4}, e\right)$ and so act from the left. Inspection of these elements shows that they can be written as powers of $\left(-\alpha_{2}, e\right)$. The group generated by them is a cyclic group of order 3 which we denote as $C_{3}^{l}$. Now it is easy to conclude that the two subgroups generate the direct product group $C_{3}^{l} \times Q^{r}$ of order 24, compare Coxeter and Moser [2] pp. 134-5, as the group of homotopies and of deck transformations for the 3-manifold $N 4$.

Table 4.2 The elements $g=\left(w_{l}, w_{r}\right)$ of the $\operatorname{group} \operatorname{deck}(N 4)=C_{3}^{l} \times Q^{r}$ in the notation of Table 6.2 .

\begin{tabular}{|l|l|}
\hline subgroup & elements \\
\hline$C_{3}^{l}$ & $\left(-\alpha_{2}, e\right),\left(\left(\alpha_{2}\right)^{2}, e\right),\left(\left(-\alpha_{2}\right)^{3}, e\right)=(e, e)$ \\
\hline$Q^{r}$ & $(e, \pm e),(e, \pm \mu),(e, \pm \nu),(e, \pm \omega)$ \\
\hline
\end{tabular}


The 24 center positions $u^{\prime}=\left(w_{l}^{-1} w_{r}\right)$ of $C_{3}^{l} \times Q^{r}$ reproduce the elements of the binary tetrahedral group Table $\mathbf{6 . 2}$.

For the projection to a $H$-invariant basis we first diagonalize the generator $-\alpha_{2} \in C_{3}^{l}$,

$$
\begin{aligned}
& -\alpha_{2}=c\left[\begin{array}{ll}
\exp \left(\frac{2 \pi i}{3}\right) & 0 \\
0 & \exp \left(-\frac{2 \pi i}{3}\right)
\end{array}\right] c^{\dagger} \\
& c=\left[\begin{array}{ll}
(1-i) \frac{-1+\sqrt{3}}{2 \sqrt{3-\sqrt{3}}} & -(1-i) \frac{1+\sqrt{3}}{2 \sqrt{3+\sqrt{3}}} \\
\frac{1}{\sqrt{3-\sqrt{3}}} & \frac{1}{\sqrt{3+\sqrt{3}}}
\end{array}\right] .
\end{aligned}
$$

Upon the coordinate transform from $u$ to $c^{\dagger} u$ we can replace the matrix $-\alpha_{2}$ by its diagonal representative. Now the projection to the identity representations of $C_{3}^{l}$ simply requires $m_{1} \rightarrow \rho \equiv 0 \bmod 3$ and excludes any other value of $m_{1}$. Next we consider the group $Q^{r}$ acting from the right. We simply transcribe the result on the group $Q$ from [8], Table 10 from left to right action. Combining left and right action into $C_{3}^{l} \times Q^{r}$ we arrive at the $H$-invariantbasis of the harmonic analysis on $N 4$ given in Table 4.3.

Table 4.3: The $\left(C_{3}^{l} \times Q^{r}\right)$-invariantbasis for the manifold $N 4$ in terms of Wigner polynomials $D^{j}$. Only integer values of $j$ appear. The coordinate transform $u \rightarrow$ $u^{\prime}=c^{\dagger} u$ in $D^{j}(u)$ follows with $c$ from eq. 13.

\begin{tabular}{|l|}
\hline$j=$ odd, $j \geq 3, m_{2}=$ even, $0<m_{2} \leq j, m_{1}=\rho \equiv 0 \bmod 3:$ \\
$\phi_{\rho, m_{2}}^{j \text { odd }}=\left[D_{\rho, m_{2}}^{j}\left(u^{\prime}\right)-D_{\rho,-m_{2}}^{j}\left(u^{\prime}\right)\right]$, \\
\hline$j=$ even, $m_{2}=0, m_{1}=\rho \equiv 0 \bmod 3:$ \\
$\phi_{\rho, 0}^{\text {jeven }}=D_{\rho, 0}^{j}\left(u^{\prime}\right)$ \\
\hline$j \geq 2$, even, $0<m_{2} \leq j, m_{2}=\operatorname{even}, m_{1}=\rho \equiv 0 \bmod 3:$ \\
\hline$\phi_{\rho, m_{2}}^{\text {jeven }}=\left[D_{\rho, m_{2}}^{j}\left(u^{\prime}\right)+D_{\rho,-m_{2}}^{j}\left(u^{\prime}\right)\right]$ \\
\hline
\end{tabular}

\section{$5 \quad$ Manifold N5}

Face gluings:

$$
F 6 \cup F 8, F 1 \cup F 4, F 2 \cup F 7, F 3 \cup F 5
$$


Edge gluing scheme:

$$
\left[\begin{array}{lll}
1 & 4 & 9 \\
2 & \overline{7} & \overline{12} \\
3 & 6 & 8 \\
5 & \overline{10} & 11
\end{array}\right]
$$

Edge and face gluing generators of $\pi_{1}(N 5)$ :

$$
\begin{aligned}
& g_{1}: 6 \cup 8,\left[\begin{array}{lll}
\overline{10} & & \overline{7} \\
& 6 &
\end{array}\right] \rightarrow\left[\begin{array}{lll}
5 & & \overline{12} \\
& 8 &
\end{array}\right], \\
& g_{2}: 1 \cup 4,\left[\begin{array}{lll}
\overline{9} & & \overline{1} \\
& \overline{2} &
\end{array}\right] \rightarrow\left[\begin{array}{lll}
\overline{1} & & \overline{4} \\
& 12 &
\end{array}\right], \\
& g_{3}: 2 \cup 7,\left[\begin{array}{lll}
2 & & \overline{3} \\
& 10 &
\end{array}\right] \rightarrow\left[\begin{array}{lll}
\overline{7} & & \overline{8} \\
& \overline{11} &
\end{array}\right], \\
& g_{4}: 3 \cup 5,\left[\begin{array}{lll}
\overline{11} & & 3 \\
& \overline{4} &
\end{array}\right] \rightarrow\left[\begin{array}{lll}
\overline{5} & & 6 \\
& \overline{9} &
\end{array}\right],
\end{aligned}
$$

Isomorphic generators of $\operatorname{deck}(N 5)$ :

$$
\begin{aligned}
& g_{1}=\left(W_{1} W_{2}\right)\left(W_{3} W_{2}\right)\left(W_{4} W_{0}\right) J_{4} \\
& g_{2}=\left(W_{2} W_{1}\right)\left(W_{4} W_{0}\right)\left(W_{2} W_{3}\right)^{2}\left(W_{2} W_{1}\right) J_{4} \\
& g_{3}=\left(W_{1} W_{2}\right)\left(W_{2} W_{3}\right)^{2}\left(W_{1} W_{2}\right)\left(W_{4} W_{0}\right)\left(W_{2} W_{3}\right)\left(W_{2} W_{1}\right) J_{4} \\
& g_{4}=\left(W_{2} W_{1}\right)\left(W_{2} W_{3}\right)^{2}\left(W_{1} W_{2}\right)\left(W_{4} W_{0}\right)\left(W_{2} W_{3}\right)^{2}\left(W_{1} W_{2}\right) J_{4}
\end{aligned}
$$

\begin{tabular}{|c|c|c|c|c|c|c|}
\hline$g$ & \multicolumn{3}{|l|}{$w_{l}$} & \multicolumn{3}{|l|}{$w_{r}$} \\
\hline \multirow[b]{2}{*}{$g_{1}$} & $\sqrt{1}$ & & & $\sqrt{1}$ & -1 & -1 \\
\hline & $\sqrt{2}$ & & $i$ & $\sqrt{2}$ & 1 & -1 \\
\hline$g_{2}$ & \multicolumn{3}{|l|}{$\alpha_{2}$} & \multicolumn{3}{|l|}{$\nu$} \\
\hline \multirow{2}{*}{$g_{3}$} & & & & $\sqrt{1}$ & & 1 \\
\hline & & 0 & & $\sqrt{2}$ & -1 & 1 \\
\hline$g_{4}$ & \multicolumn{3}{|l|}{$\alpha_{2}$} & \multicolumn{3}{|l|}{$\bar{e}$} \\
\hline
\end{tabular}

Table 5.1 Generators $g=\left(w_{l}, w_{r}\right)$ of $\operatorname{deck}(N 5)$ with partial use of Table 6.2. Note that the matrices $\left(w_{l}, w_{r}\right)$ for the generators $g_{1}, g_{3}$ do not occur in Table 6.2 and so do not belong to the binary tetrahedral group.

Table 5.2 Elements $g_{j}=\left(w_{l}, w_{r}\right), j= \pm 1, \ldots, \pm 12$ of the group $\operatorname{deck}(N 5)$, enumerated according to the 24 octahedral center positions $u^{\prime}=w_{l}^{-1} w_{r} \in S^{3}$, in the order 
and notation of Table 6.2 .

\begin{tabular}{|c|c|c|c|c|c|}
\hline $\pm j$ & \multicolumn{2}{|l|}{$w_{l}$} & \multicolumn{2}{|l|}{$w_{r}$} & $w_{l}^{-1} w_{r}$ \\
\hline \pm 1 & \multicolumn{2}{|l|}{$\alpha_{2}^{-1}$} & \multicolumn{2}{|l|}{$\mp \nu$} & $\pm \alpha_{1}$ \\
\hline \pm 2 & \multicolumn{2}{|l|}{$\alpha_{2}^{-1}$} & \multicolumn{2}{|l|}{ $\pm e$} & $\pm \alpha_{2}$ \\
\hline \pm 3 & \multicolumn{2}{|l|}{$\alpha_{2}$} & \multicolumn{2}{|l|}{$\pm \nu$} & $\pm \alpha_{3}$ \\
\hline \pm 4 & $\sqrt{\frac{1}{2}}$ & $\begin{array}{ll}-i & -1 \\
1 & i\end{array}$ & $\pm \sqrt{\frac{1}{2}}$ & $\begin{array}{ll}1 & -1 \\
1 & 1\end{array}$ & $\pm \alpha_{4}$ \\
\hline \pm 5 & $\sqrt{\frac{1}{2}}$ & $\begin{array}{ll}-i & -1 \\
1 & i\end{array}$ & $\mp \sqrt{\frac{1}{2}}$ & $\begin{array}{ll}1 & 1 \\
-1 & 1\end{array}$ & $\pm \alpha_{1}^{-1}$ \\
\hline \pm 6 & \multicolumn{2}{|l|}{$\alpha_{2}$} & \multicolumn{2}{|l|}{ $\pm e$} & $\pm \alpha_{2}^{-1}$ \\
\hline \pm 7 & $\begin{array}{l}0 \\
-\theta\end{array}$ & $\begin{array}{l}\bar{\theta} \\
0\end{array}$ & $\mp \sqrt{\frac{1}{2}}$ & $\begin{array}{ll}1 & -1 \\
1 & 1\end{array}$ & $\pm \alpha_{3}^{-1}$ \\
\hline \pm 8 & & $\begin{array}{l}\bar{\theta} \\
0\end{array}$ & $\pm \sqrt{\frac{1}{2}}$ & $\begin{array}{ll}1 & 1 \\
-1 & 1\end{array}$ & $\pm \alpha_{4}^{-1}$ \\
\hline \pm 9 & \multicolumn{2}{|l|}{$e$} & \multicolumn{2}{|l|}{ $\pm e$} & $\pm e$ \\
\hline \pm 10 & $-\sqrt{\frac{1}{2}}$ & $\begin{array}{ll}i & i \\
i & -i\end{array}$ & $\pm \sqrt{\frac{1}{2}}$ & $\begin{array}{ll}1 & 1 \\
-1 & 1\end{array}$ & $\pm \mu$ \\
\hline \pm 11 & $e$ & & $\pm \nu$ & & $\pm \nu$ \\
\hline \pm 12 & $\sqrt{\frac{1}{2}}$ & $\begin{array}{ll}i & i \\
i & -i\end{array}$ & $\pm \sqrt{\frac{1}{2}}$ & $\begin{array}{ll}1 & -1 \\
1 & 1\end{array}$ & $\pm \omega$ \\
\hline
\end{tabular}

\section{Manifold N6}

Face gluings:

$F 6 \cup F 4, F 5 \cup F 3, F 8 \cup F 2, F 7 \cup F 1$.

Edge gluing scheme:

$$
\left[\begin{array}{lll}
1 & 8 & 10 \\
2 & 5 & 11 \\
3 & 6 & 12 \\
4 & 7 & 9
\end{array}\right]
$$

Edge and face gluing generators of $\pi_{1}(N 6)$ :

$$
\begin{aligned}
& g_{1}: 6 \cup 4,\left[\begin{array}{lll}
\overline{10} & & \overline{7} \\
& 6 &
\end{array}\right] \rightarrow\left[\begin{array}{lll}
\overline{1} & & \overline{4} \\
& 12 &
\end{array}\right], \\
& g_{2}: 5 \cup 3,\left[\begin{array}{lll}
5 & & 9 \\
& \overline{6} &
\end{array}\right], \rightarrow\left[\begin{array}{lll}
11 & & 4 \\
& \overline{3} &
\end{array}\right], \\
& g_{3}: 8 \cup 2,\left[\begin{array}{llll}
12 & & \overline{5} \\
& \overline{8} &
\end{array}\right] \rightarrow\left[\begin{array}{lll}
3 & & \overline{2} \\
& \overline{10} &
\end{array}\right],
\end{aligned}
$$




$$
g_{4}: 7 \cup 1,\left[\begin{array}{lll}
7 & & 11 \\
& 8 &
\end{array}\right] \rightarrow\left[\begin{array}{lll}
9 & & 2 \\
& 1 &
\end{array}\right] .
$$

Isomorphic generators of $\operatorname{deck}(N 6)$ :

$$
\begin{aligned}
& g_{1}=\left(W_{1} W_{2}\right)\left(W_{4} W_{0}\right) J_{4}, \\
& g_{2}=\left(W_{3} W_{2}\right)\left(W_{1} W_{2}\right)\left(W_{4} W_{0}\right)\left(W_{2} W_{3}\right) J_{4}, \\
& g_{3}=\left(W_{2} W_{3}\right)^{2}\left(W_{1} W_{2}\right)\left(W_{4} W_{0}\right)\left(W_{2} W_{3}\right)^{2} J_{4}, \\
& g_{4}=\left(W_{2} W_{3}\right)\left(W_{1} W_{2}\right)\left(W_{4} W_{0}\right)\left(W_{3} W_{2}\right) J_{4},
\end{aligned}
$$

Table 6.1 Generators $g=\left(w_{l}, w_{r}\right)$ of $\operatorname{deck}(N 6)$, compare Table 6.2.

\begin{tabular}{|l|l|l|}
\hline$g$ & $w_{l}$ & $w_{r}$ \\
\hline$g_{1}$ & $\sqrt{\frac{1}{2}}\left[\begin{array}{ll}\theta & \theta \\
-\bar{\theta} & \bar{\theta}\end{array}\right]:=\alpha_{1}$ & $e$ \\
\hline$g_{2}$ & $\sqrt{\frac{1}{2}}\left[\begin{array}{ll}\bar{\theta} & \theta \\
-\bar{\theta} & \theta\end{array}\right]:=\alpha_{2}^{-1}$ & $e$ \\
\hline$g_{3}$ & $\sqrt{\frac{1}{2}}\left[\begin{array}{ll}\bar{\theta} & -\bar{\theta} \\
\theta & \theta\end{array}\right]:=\alpha_{4}^{-1}$ & $e$ \\
\hline$g_{4}$ & $\sqrt{\frac{1}{2}}\left[\begin{array}{ll}\theta & -\bar{\theta} \\
\theta & \bar{\theta}\end{array}\right]:=\alpha_{3}$ & $e$ \\
\hline
\end{tabular}

Using the equivalence $\left(g_{l}, g_{r}\right) \sim\left(-g_{l},-g_{r}\right)$, we can write $H$ entirely in terms of left actions. The group $H$ of homotopies and deck transformations of the 3-manifold $N 6$ then turns out to be the binary tetrahedral group $<2,3,3>$ of order 24 in the notation of Coxeter and Moser [2] pp. 134-5. The elements and multiplication rules are given in Tables 6.2, 6.3.

Table 6.2: The binary tetrahedral group $\mathcal{T} \sim \operatorname{deck}(N 6)$ has 16 elements $\pm \alpha_{j}, \pm \alpha_{j}^{-1}$ and 8 elements $\pm e, \pm \mu, \pm \nu, \pm \omega$, with $\theta=\exp (i \pi / 4), \bar{\theta}=\exp (-i \pi / 4)$. It acts from 
the left on $u \in S^{3}$.

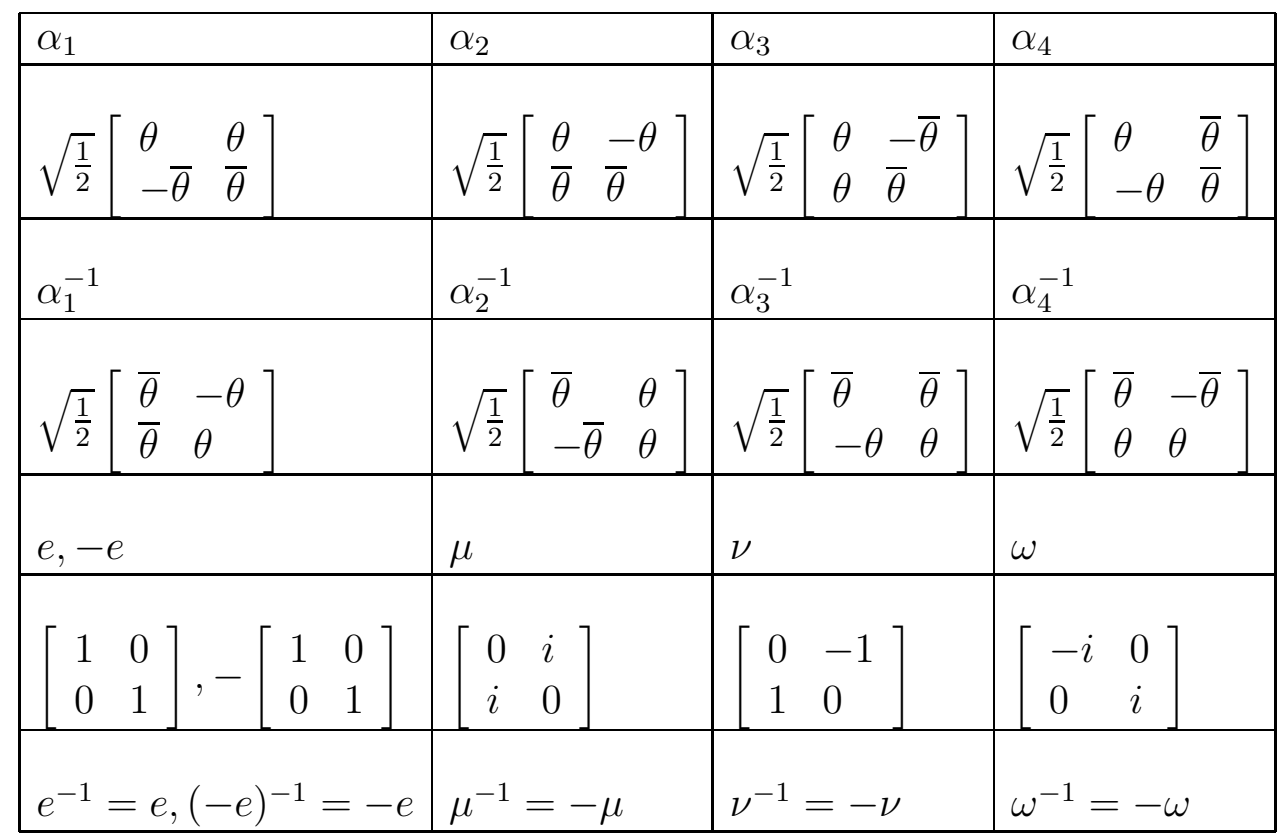

The elements in this Table obey

$$
\begin{aligned}
& \left(\alpha_{j}\right)^{3}=\left(\alpha_{j}\right)^{-3}=-e, \frac{1}{2} \operatorname{Tr}\left(\alpha_{j}\right)=\frac{1}{2} \operatorname{Tr}\left(\alpha_{j}^{-1}\right)=\frac{1}{2}, j=1, . ., 4 . \\
& \mu^{2}=\nu^{2}=\omega^{2}=-e
\end{aligned}
$$

The last four elements generate as subgroup the quaternion group $Q$ of order 8 with $\mathbf{i}=-\omega, \mathbf{j}=-\nu, \mathbf{k}=\mu$. 
Table 6.3 Multiplication table for 12 elements $g$ of the binary tetrahedral group $\operatorname{deck}(N 6)$ given in Table 6.2. The 12 elements $-g$ have been suppressed.

\begin{tabular}{|l|llll|llll|llll|}
\hline & $\alpha_{1}$ & $\alpha_{2}$ & $\alpha_{3}$ & $\alpha_{4}$ & $\alpha_{1}^{-1}$ & $\alpha_{2}^{-1}$ & $\alpha_{3}^{-1}$ & $\alpha_{4}^{-1}$ & $\mu$ & $\nu$ & $\omega$ & $e$ \\
\hline$\alpha_{1}$ & $-\alpha_{1}^{-1}$ & $\alpha_{4}$ & $-\omega$ & $-\nu$ & $e$ & $\mu$ & $\alpha_{2}^{-1}$ & $\alpha_{3}$ & $-\alpha_{3}^{-1}$ & $\alpha_{2}$ & $\alpha_{4}^{-1}$ & $\alpha_{1}$ \\
$\alpha_{2}$ & $\alpha_{3}$ & $-\alpha_{2}^{-1}$ & $\nu$ & $-\omega$ & $-\mu$ & $e$ & $\alpha_{4}$ & $\alpha_{1}^{-1}$ & $\alpha_{4}^{-1}$ & $-\alpha_{1}$ & $\alpha_{3}^{-1}$ & $\alpha_{2}$ \\
$\alpha_{3}$ & $\mu$ & $-\omega$ & $-\alpha_{3}^{-1}$ & $\alpha_{1}$ & $\alpha_{2}$ & $\alpha_{4}^{-1}$ & $e$ & $\nu$ & $-\alpha_{4}$ & $-\alpha_{2}^{-1}$ & $\alpha_{1}^{-1}$ & $\alpha_{3}$ \\
$\alpha_{4}$ & $-\omega$ & $-\mu$ & $\alpha_{2}$ & $-\alpha_{4}^{-1}$ & $\alpha_{3}^{-1}$ & $\alpha_{1}$ & $-\nu$ & $e$ & $\alpha_{3}$ & $\alpha_{1}^{-1}$ & $\alpha_{2}^{-1}$ & $\alpha_{4}$ \\
\hline$\alpha_{1}^{-1}$ & $e$ & $\nu$ & $\alpha_{4}^{-1}$ & $\alpha_{2}$ & $-\alpha_{1}$ & $\alpha_{3}^{-1}$ & $-\mu$ & $\omega$ & $\alpha_{2}^{-1}$ & $-\alpha_{4}$ & $-\alpha_{3}$ & $\alpha_{1}^{-1}$ \\
$\alpha_{2}^{-1}$ & $-\nu$ & $e$ & $\alpha_{1}$ & $\alpha_{3}^{-1}$ & $\alpha_{4}^{-1}$ & $-\alpha_{2}$ & $\omega$ & $\mu$ & $-\alpha_{1}^{-1}$ & $\alpha_{3}$ & $-\alpha_{4}$ & $\alpha_{2}^{-1}$ \\
$\alpha_{3}^{-1}$ & $\alpha_{4}$ & $\alpha_{1}^{-1}$ & $e$ & $-\mu$ & $\omega$ & $-\nu$ & $-\alpha_{3}$ & $\alpha_{2}^{-1}$ & $\alpha_{1}$ & $\alpha_{4}^{-1}$ & $-\alpha_{2}$ & $\alpha_{3}^{-1}$ \\
$\alpha_{4}^{-1}$ & $\alpha_{2}^{-1}$ & $\alpha_{3}$ & $\mu$ & $e$ & $\nu$ & $\omega$ & $\alpha_{1}^{-1}$ & $-\alpha_{4}$ & $-\alpha_{2}$ & $-\alpha_{3}^{-1}$ & $-\alpha_{1}$ & $\alpha_{4}^{-1}$ \\
\hline$\mu$ & $-\alpha_{2}$ & $\alpha_{1}$ & $-\alpha_{1}^{-1}$ & $\alpha_{2}^{-1}$ & $\alpha_{3}$ & $-\alpha_{4}$ & $\alpha_{4}^{-1}$ & $-\alpha_{3}^{-1}$ & $-e$ & $-\omega$ & $\nu$ & $\mu$ \\
$\nu$ & $\alpha_{4}^{-1}$ & $-\alpha_{3}^{-1}$ & $-\alpha_{4}$ & $\alpha_{3}$ & $-\alpha_{2}^{-1}$ & $\alpha_{1}^{-1}$ & $\alpha_{2}$ & $-\alpha_{1}$ & $\omega$ & $-e$ & $-\mu$ & $\nu$ \\
$\omega$ & $\alpha_{3}^{-1}$ & $\alpha_{4}^{-1}$ & $\alpha_{2}^{-1}$ & $\alpha_{1}^{-1}$ & $-\alpha_{4}$ & $-\alpha_{3}$ & $-\alpha_{1}$ & $-\alpha_{2}$ & $-\nu$ & $\mu$ & $-e$ & $\omega$ \\
$e$ & $\alpha_{1}$ & $\alpha_{2}$ & $\alpha_{3}$ & $\alpha_{4}$ & $\alpha_{1}^{-1}$ & $\alpha_{2}^{-1}$ & $\alpha_{3}^{-1}$ & $\alpha_{4}^{-1}$ & $\mu$ & $\nu$ & $\omega$ & $e$ \\
\hline
\end{tabular}

\section{Conclusion.}

In the present work we extend the study of the harmonic analysis on Platonic 3manifolds beyond the dodecahedral, the tetrahedral and the two cubic spherical manifolds. From homotopy we construct and identify three groups $H,|H|=24$ of deck transformations for three octahedral spherical 3-manifolds and give their action on the 3-sphere. Representation theory of $S O(4, R)>H$ provides the tools for the multiplicity and projection of $H$-invariant polynomial bases of the harmonic analysis on the octahedral 3-manifolds.

\section{Acknowledgment.}

The author appreciates the assistance of T Kramer, Institute for Theoretical Physics, University of Regensburg, Germany, who did the essential algebraic computations for the Tables. He is indebted to B Everitt, Dept. of Mathematics, University of York, England, UK, for references, in particular [1].

\section{References}

[1] Cavicchioli A, Spaggiari F, and Tellone A I, Topology of compact space forms from Platonic solids.I. Topology and its Applications 156 (2009), pp. 812-822 
[2] Coxeter H S M and Moser W O J, Generators and relations for discrete groups. Springer, Berlin 1965

[3] Everitt B, 3-manifolds from Platonic solids. Topology and its Applications 138 (2004), 253-63

[4] Humphreys J E, Reflection groups and Coxeter groups. Cambridge University Press, Cambridge 1990

[5] Kramer P, An invariant operator due to F Klein quantizes H Poincare's dodecahedral manifold. J Phys A: Math Gen 38 (2005) 3517-40

[6] Kramer P, Harmonic polynomials on the Poincare dodecahedral 3-manifold. J. of Geometry and Symmetry in Physics 6 (2006) 55-66

[7] Kramer P, Platonic polyhedra tune the 3-sphere: Harmonic analysis on simplices. Physica Scripta 79 (2009) 045008, arXiv:0810.3403

[8] Kramer P, Platonic polyhedra tune the 3-sphere II: Harmonic analysis on cubic spherical 3-manifolds. Physica Scripta 80 (2009) 025902, arXiv:0901.0511

[9] Kramer P, Platonic topology and CMB fluctuation: Homotopy, anisotropy, and multipole selection rules. submitted for publication, arXiv:0909.2758

[10] Lachieze-Rey M and Luminet J-P, Cosmic topology. Phys Rep 254 (1995) 135214

[11] Luminet J-P, Weeks J R, Riazuelo A, Lehoucq R and Uzan J-Ph, Dodecahedral space topology as an explanation for weak wide-angle temperature correlations in the cosmic microwave background. Nature 425 (2003) 593-5

[12] Seifert H and Threlfall W, Lehrbuch der Topologie. Leipzig 1934, Chelsea Reprint, New York 1980

[13] Sommerville D M Y, An introduction to the geometry of $N$ dimensions. Dover, New York 1958

[14] Thurston W P, Three-Dimensional Geometry and Topology. Princeton University Press, Princeton 1997 